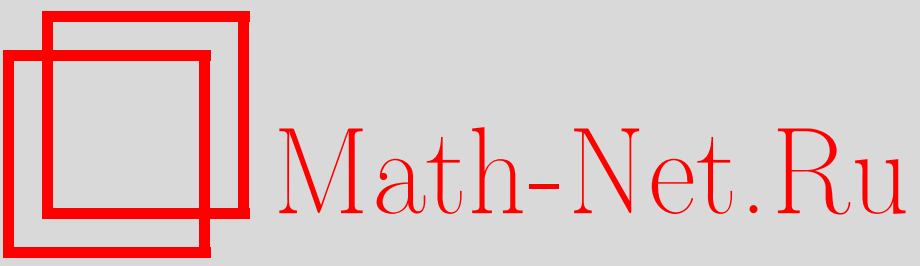

П. А. Колгушкин, Классификация простых мультиростков кривых в контактном пространстве, УМH, 2004, том 59, выпуск 3, 171-172

DOI: https://doi.org/10.4213/rm749

Использование Общероссийского математического портала Math-Net.Ru подразумевает, что вы прочитали и согласны с пользовательским соглашением

http://www.mathnet.ru/rus/agreement

Параметры загрузки:

IP: 54.210 .77 .194

26 апреля 2023 г., 17:43:52 


\title{
КЛАССИФИКАЦИЯ ПРОСТЫХ МУЛЬТИРОСТКОВ КРИВЫХ В КОНТАКТНОМ ПРОСТРАНСТВЕ
}

\author{
П. А. Колгушкин
}

Ростком приводимой кривой (или мультиростком) в начале координат в пространстве $\mathbb{C}^{N}$ называется набор $F=\left(f_{1}, \ldots, f_{k}\right)$ ростков аналитических отображений $f_{i}:(\mathbb{C}, 0) \rightarrow\left(\mathbb{C}^{N}, 0\right)$, где $\operatorname{Im} f_{i} \cap \operatorname{Im} f_{j}=\{0\}$ для $i \neq j\left(f_{1}, \ldots, f_{k}\right.$ называются компонентами мульиростка). Мультиросток, состоящий из одной компоненты, называется неприводимым или просто ростком.

Пусть $R$ - группа координатных замен в $(\mathbb{C}, 0)$, т.е. группа ростков невьрожденных аналитических отображений $(\mathbb{C}, 0) \rightarrow(\mathbb{C}, 0)$. Предположим, что $N=2 n+1$ и в начале координат в пространстве $\mathbb{C}^{2 n+1}$ задан росток контактной структуры, т.е. распределения гиперплоскостей в касателшном расслоении, удовлетворяющего условию "максималшной неинтегрируемости". Пусть Cont - подгруппа группы координатных замен в пространстве $\left(\mathbb{C}^{2 n+1}, 0\right)$, состоящая из ростков контактных диффеоморфизмов, т.е. диффеоморфизмов, сохраняющих контактную структуру. Группа $R_{(1)} \times \cdots \times R_{(k)} \times$ Cont (здесь $R_{(i)}-$ экземпляр группы $R$ с номером $i$ ) действует на пространстве мультиростков, содержащих $k$ компонент, по формуле

$$
\left(g, h_{1}, \ldots, h_{k}\right) \cdot\left(f_{1}, \ldots, f_{k}\right)=\left(g \circ f_{1} \circ h_{1}^{-1}, \ldots, g \circ f_{k} \circ h_{k}^{-1}\right) .
$$

Мультиросток $F=\left(f_{1}, \ldots, f_{k}\right)$ назьвается (контактно) простым, если в пространстве мультиростков найдется окрестность, содержащая $F$ и пересекающая только конечное число орбит указанного действия. Мултиросток назьвается стабильн простьм, если он остается простьм после вложения $\mathbb{C}^{2 n+1} \hookrightarrow \mathbb{C}^{2 N+1}$, причем это вложение должно быть согласовано с контактными структурами. Мультиростки, эквивалентные после таких вложений, назьваются (контактно) стабильно эквивалентными.

В работе [1] была описана особенность $A_{2}$ (т.е. особенность $\left.\left(t^{2}, t^{3}, 0, \ldots, 0\right)\right)$ в контактном пространстве в том случае, когда она является простой. В настоящей заметке приведена классификация стабильно простых особенностей мультиростков в контактном пространстве относителшно формальной стабильной эквивалентности (т.е. правые и левые замены задаются формальными степенными рядами).

Предположим, что в $\mathbb{C}^{2 n+1}$ задана система координат $\left(z, q_{1}, \ldots, q_{n}, p_{1}, \ldots, p_{n}\right)$, в которых форма, задающая контактную структуру, имеет стандартньй вид $d z+\sum_{i=1}^{n} p_{i} d q_{i}$. Точку с такими координатами мы будем обозначать $\left(z \mid q_{1}, \ldots, q_{n} ; p_{1}, \ldots, p_{n}\right)$. Если координаты $p_{i}$ и $q_{i}$ равны нулю для всех $i>i_{0}$, то иногда при записи эти координаты мы будем опускать.

ТеОРема. Всякий (контактно) стабильно простой мультиросток с точностью до перестановки компонент стабильно формально әквивалентен одному и только одному мультиростку из следующего списка.

\section{I. Особенности неприводимых кривых}

\section{I.1. Регулярные кривые.}

\section{I.2. Кривые кратности 2.}

$$
\text { 1. }(t \mid 0 ; 0) \quad \text { 2. }\left(t^{m} \mid t ; 0\right), \quad m>1
$$
1. $\left(t^{3} \mid t^{2} ; 0\right)$
2. $\left(t^{2} \mid t^{2 m+1} ; 0\right)$
3. $\left(t^{4}+t^{2 m+1} \mid t^{2} ; 0\right)$
4. $\left(t^{4} \mid t^{2} ; t^{2 m+1}\right)$
5. $\left(t^{4} \mid t^{2}, t^{2 m+1} ; 0,0\right)$

I.3. Кривые кратности 3.
1. $\left(t^{3} \mid t^{4} ; t^{5}\right)$
2. $\left(t^{3} \mid t^{4}, t^{5} ; 0,0\right)$
3. $\left(t^{3} \mid t^{4}+t^{5} ; 0\right)$
4. $\left(t^{3} \mid t^{4} ; 0\right)$
5. $\left(t^{3} \mid t^{5} ; t^{7}\right)$
6. $\left(t^{3} \mid t^{5}, t^{7} ; 0,0\right)$
7. $\left(t^{3} \mid t^{5}+t^{7} ; 0\right)$
8. $\left(t^{3} \mid t^{5} ; 0\right)$
9. $\left(t^{3} \mid t^{7} ; t^{8}\right)$
10. $\left(t^{3} \mid t^{7}, t^{8} ; t^{11}, 0\right)$
11. $\left(t^{3} \mid t^{7}, t^{8} ; 0,0\right)$
12. $\left(t^{4} \mid t^{3} ; t^{5}\right)$
13. $\left(t^{4} \mid t^{3}, t^{5} ; 0,0\right)$
14. $\left(t^{4}+t^{6} \mid t^{3} ; 0\right)$
15. $\left(t^{4} \mid t^{3} ; 0\right)$ 


\section{II. Особенности приводимых кривых}

\section{II.1. Мультростки, состоящие из неособых компонент.}

II.1.1. Мультиростки, состоящие из двух неособых компонент. Первая компонента имеет вид $(t \mid 0 ; 0)$. Мы указьваем вторую компоненту.
1. $(t \mid t ; 0)$
2. $\left(t^{m} \mid t ; 0\right), \quad m>1$
3. $\left(t \mid t^{m} ; 0\right)$

II.1.2. Мультиростки, состоящие из трех неособых компонент. Первые две компоненты имеют вид $((t \mid 0,0 ; 0,0),(t \mid t, 0 ; 0,0))$. Мы указываем третью компоненту.
1. $(t \mid 0 ; t)$
2. $(t \mid 0, t ; 0,0)$
3. $\left(t^{m} \mid 0 ; t\right), \quad m>1$
4. $\left(t^{m} \mid 0, t ; 0,0\right), \quad m>1$

II.2. Мультиростки, содержащие одну неособую и одну особую компоненту. Неособая компонента имеет вид $(t \mid 0,0 ; 0,0)$. Мы указьваем особую компоненту.
1. $\left(t^{2} \mid t^{2} ; t^{2 m+1}\right)$
2. $\left(t^{2} \mid t^{2}, t^{2 m+1} ; 0,0\right)$
3. $\left(t^{2} \mid t^{2}+t^{2 m+1} ; 0\right)$
4. $\left(t^{3} \mid t^{2} ; t^{3}\right)$
5. $\left(t^{3} \mid t^{2}, t^{3} ; 0,0\right)$
6. $\left(t^{3} \mid t^{2}+t^{3} ; 0\right)$
7. $\left(t^{3} \mid t^{2} ; 0\right)$
8. $\left(t^{2} \mid t^{3} ; t^{4}\right)$
9. $\left(t^{2} \mid t^{3}, t^{4} ; 0,0\right)$
10. $\left(t^{2} \mid t^{3}+t^{4} ; 0\right)$
11. $\left(t^{2} \mid t^{3} ; 0\right)$
12. $\left(t^{2} \mid t^{4}, 0 ; t^{5}, 0\right)$
13. $\left(t^{2} \mid t^{4}, t^{5} ; t^{7}, 0\right)$
14. $\left(t^{2} \mid t^{4}, t^{5} ; 0,0\right)$

Доказательства основаны на гомотопическом методе и классификации простых особенностей мультиростков в симплектическом пространстве, полученной в [2].

Из доказательства этой теоремы следует, что класс мультиростков, простых с контактной точки зрения, совпадает с классом стабильно простых мультиростков (с контактной точки зрения).

\section{СПИСОК ЛИТЕРАТУРЫ}

[1] V. I. Arnold // Canad. J. Math. 1999. V. 51. №6. Р. 1123-1134. [2] П. А. Колгушкин // Алгебра и анализ. 2003. Т. 15. № 1. С. 148-183.

Принято редколлегией 01.04 .2004 